\title{
Ancient Whole Grain Gluten-Free Flatbreads
}

\author{
Talwinder Singh Kahlon, Mei-Chen Maggie Chiu \\ Western Regional Research Center, USDA-ARS, Albany, USA \\ Email: Talwinder.kahlon@ars.usda.gov
}

Received 9 July 2014; revised 10 August 2014; accepted 18 August 2014

Copyright (C) 2014 by authors and Scientific Research Publishing Inc.

This work is licensed under the Creative Commons Attribution International License (CC BY). http://creativecommons.org/licenses/by/4.0/

(c) (i) Open Access

\section{Abstract}

This is the only report demonstrating innovative ancient whole grain gluten-free (no yeast or chemicals) products. Ancient whole grain gluten-free flatbreads were prepared with quinoa, teff, amaranth and buckwheat flours. Dough formulations contained flour, salt and water. Ingredients were mixed for 5 min using table top Kitchen Aid mixer at stir setting 1. Dough was equilibrated for $30 \mathrm{~min} .65 \mathrm{~g}$ of dough was placed between two sheets of nonstick parchment paper and pressed to $17 \mathrm{~cm}$ round flatbreads in a Tortilla Chapatti Press. Each flatbread was cooked for two minutes (one minute each side) in using Flatbread Maker. Taste panels of 64 in-house volunteers determined that Taste/Flavor of quinoa, teff and amaranth flatbreads were similar and significantly $(P \leq 0.05)$ better than those for buckwheat flatbread. The taste panel determined ancient whole grain gluten-free flatbreads had acceptance of quinoa $84 \%$, teff $72 \%$, amaranth $66 \%$ and buckwheat $38 \%$. Each ancient whole grain gluten-free flatbread contained $25-30 \mathrm{~g}$ whole grain and 4 - 5 g protein. Quinoa, teff and amaranth one flatbread contained 2 - $3 \mathrm{~g}$ dietary fiber, whereas buckwheat flatbread contained $8 \mathrm{~g}$ dietary fiber. Consuming two whole grain gluten-free flatbreads with two meals (total $=4$ ) would give 4 - $32 \mathrm{~g}$ of dietary fiber. The USDA food guide recommends that at least $1 / 2$ of all the grains eaten should be whole grains. The FDA allows food Health Claim labels for food containing $51 \%$ whole gains and $11 \mathrm{~g}$ of dietary fiber. The gluten sensitive individuals would enjoy tasty, health promoting, ancient whole grain easy to make flatbreads. These recipes offer consumers additional nutritious gluten-free choices and would lead to increase in whole grain consumption.

\section{Keywords}

Ancient Whole Grains, Gluten-Free, Flatbreads, Quinoa, Teff, Amaranth, Buckwheat

\section{Introduction}

Well over 6000 years people have been making flatbreads. The oldest baker's oven in the world was known in Babylon in 4000 BC. Flatbreads were baked in hot ashes or on heated stone slabs in the old kingdom of Egypt as long ago as 2500 BC. Currently over 1.8 billion people worldwide eat traditional flatbreads. Flatbreads are in 
general round, flat, unleavened breads (no yeast or chemicals). Flatbreads are called by various names in the world such as Arepa, Bazlama, Chapatti, Flatbrod, Flatkaka, Focaccia, Khubz, Injera, Laobing, Lavash, Lefse, Matzoh, Naan, Pita, Paratha, Phulka, Puri, Roti, Tortilla Tunnbrod and Yufka. Pancakes, crepes and pizza are also flatbreads that contain baking powder or eggs or yeast. Other food ingredients such as meats, vegetables, condiments or spices may be incorporated into the dough. Flatbreads could be baked or fried to make snacks such as chips. Sandwiches, toasts, wraps or roll-ups are made using flatbreads. Flatbreads are served with soups, chutnies, curries, pickles, vegetables and meat dishes. Flatbreads can be used for scooping, dipping or wrapping around a variety food dishes. Consuming two flatbreads in a meal for breakfast, lunch and dinner is quite common in flatbread consuming cultures. With the changing demographics and ethnic character of the world population, various kinds of flatbreads are increasing in popularity. Recently many of the fast food chains have success in introducing flatbread wraps. Wheat is the common ingredient of most flatbreads. Wheat flatbreads made on a griddle "Chapatti, Phulka or Roti”, oven baked "Naan", pan fried "Paratha". Qarooni, et al. [1] reviewed the flat breads of the world. USDA, Center for Nutrition Policy and Promotion; Dietary Guidelines for Americans, http://www.choosemyplate.gov/ [2], recommends that, at least $1 / 2$ of all the grains eaten should be whole grains. It is recommended that whole grains should be used in making food products rather than refined grains. Whole Grain Council http://www.wholegrainscouncil.org http://wholegrainscouncil.org/whole-grains-101/what-are-the-health-benefits [3] has summarized the scientific studies of the health benefits of whole grains. It has been documented that eating whole grains instead of refined grains lowers the risk of many chronic diseases. While benefits are most pronounced for those consuming at least 3 servings daily, some studies show reduced risks from as little as one serving daily. US Food and Drug Administration

http://google2.fda.gov/search?q=whole+grain+claim\&client=FDAgov\&site=FDAgov\&lr=\&proxystylesheet=F DAgov\&requiredfields=-archive\%3AYes\&output $=x m l$ no_dtd\&getfields $=*$ [4] allows label health claim for food containing $51 \%$ whole grains and $11 \mathrm{~g}$ of dietary fiber. Whole grains are $100 \%$ extraction flours. Whole grains flours contain the entire grain kernel (bran, germ, and endosperm). Wheat is the main grain of the bread loafs and flatbreads due to special properties of gluten. However there is increasing evidence of hyper-sensitivity to gluten in the celiac patients. The US Food and Drug Administration (FDA) has published on August 1, 2013; a new regulation defining the term "gluten-free" for voluntary food labeling.

http://www.fda.gov/downloads/ForConsumers/ConsumerUpdates/UCM363276.pdf [5].

FDA has set a gluten limit of less than 20 ppm (parts per million) in foods that carry the label "gluten-free, free of gluten, without gluten or no gluten". This is the lowest level that can be consistently detected in foods using valid scientific analytical tools. Most people with celiac disease can tolerate foods with very small amounts $(20 \mathrm{ppm})$ of gluten. This level is consistent with those set by other countries and international bodies that set food safety standards. It provides a uniform standard definition to help up to 3 million Americans who have celiac disease. The celiac disease is an autoimmune digestive condition that can be effectively managed only by eating a gluten free diet. The celiac disease occurs when the body's natural defense system reacts to gluten by attacking the lining of the small intestine. Without a healthy intestinal lining, the body cannot absorb the nutrients it needs. Delayed growth and nutrient deficiencies can result and may lead to conditions such as anemia and osteoporosis. Other serious health problems may include diabetes, autoimmune thyroid disease and intestinal cancers. Before this FDA rule there were no federal standards or definitions for the food industry to use in labeling products "gluten-free". An estimated 5 percent of foods currently labeled "gluten-free" contain higher than $20 \mathrm{ppm}$ of gluten. Previously desirable acceptance of whole grain gluten-free flatbreads of whole grain corn, brown rice, millet and sorghum have been reported [6]. The object of the study reported herein was to offer every house hold the option to make fresh ancient whole grain gluten-free flatbreads and give family additional choices to increase the whole grains consumption in every meal. Ancient whole grains that do not contain gluten were used in order to offer healthy choice to gluten sensitive individuals. The preference was to use ancient whole grains which are easily available at a reasonable price. No gluten containing control was used as slight contamination (>20 ppm) of gluten would compromise gluten free processing. In this study, flatbreads using gluten-free ancient whole grain quinoa, teff, amaranth and buckwheat were evaluated.

\section{Methods and Materials}

Ancient whole grain flours of quinoa, teff, amaranth and buckwheat were obtained from local whole foods market. Composition of the ancient whole grain gluten-free flatbreads ingredients is given in Table 1. Samples were 
Table 1. Composition of ancient whole grain gluten-free flours (DM) basis, \%.

\begin{tabular}{cccccccc}
\hline Ingredient & Protein & Fat & Minerals & Carbohydrate & TDF & DM \\
\hline Quinoa & 16.46 & 6.01 & 3.38 & 74.15 & 8.51 & 89.25 \\
Teff & 13.81 & 3.73 & 2.86 & 79.60 & 7.71 & 89.18 \\
Amaranth & 17.16 & 7.37 & 2.74 & 72.73 & 8.81 & 90.31 \\
Buckwheat & 19.29 & 3.72 & 3.32 & 73.67 & 31.81 & 89.41 \\
\hline
\end{tabular}

Nitrogen to protein factors used was 6.25. TDF: Total Dietary Fiber; DM: Dry Matter. Carbohydrate $=[100-($ Protein + Fat + Ash $)]$.

analyzed for nitrogen by AOAC method 990.03 [7], with a Leco True Spec Nitrogen Analyzer (Leco Corp., St Joseph, MI), total dietary fiber by AOAC method 985.29 [7], crude fat with petroleum ether by accelerated solvent extractor (ASE 350 Dionex Corp., Bannockburn, IL), ash by AOAC method 942.05 [8], and moisture by AOAC method 935.29 [8]. Composition of flatbread dough is given in Table 2. Ancient whole grain flour and salt were mixed in a large bowl. Water was added slowly to make dough in a Kitchen Aid ProLine, mixing time 5 min., stir setting 1 (KitchenAid Inc., St. Joseph, Michigan, USA). The dough was covered and set at room temperature for $30 \mathrm{~min}$. Small portions (about $65 \mathrm{~g}$ ) of dough were put on a parchment paper (non-stick, oven safe) and pressed to about $17 \mathrm{~cm}$ circle in a $20 \mathrm{~cm}$ Sol Luna Tortilla Flatbread Press (Figure 1). Dough could be prepared by hand mixing and flatbreads could be made into a circle by pressing the dough with moist hands or using a rolling pin. Uncooked ancient grain flatbreads are shown in Figure 2, (A) quinoa; (B) teff; (C) amaranth; and (D) buckwheat. Flat breads were cooked for 2 minutes (1 min each side) in parchment paper on a 1000 Watts CucinaPro Flatbread Maker (www.CucinaPro.com, Figure 3). Cooked ancient grain flatbreads are shown in Figure 4 (A) quinoa; (B) teff; (C) amaranth; and (D) buckwheat. For crispier flatbreads cooking time can be adjusted as appropriate according to the personal preference. Cooked flatbreads were stored in an insulated lunch box. Flatbreads were cut into 8 sectors with a pizza cutter and all four kinds of flat bread samples were presented to the volunteer taste panel Figure 5 (A) quinoa; (B) teff; (C) amaranth; and (D) buckwheat. Tasters were given choice of tasting gluten-free flatbreads as such or use fresh mild salsa obtained from refrigerated section of a grocery store or laboratory kitchen made chutney (Figure 6). Composition of mint-cilantro-onion chutney is given in Table 3. The tasters, $\mathrm{n}=64$; evaluated the gluten-free flatbreads for Color/Appearance, Odor/Aroma, Taste/Flavor, Texture/Mouth-feel on a scale of 1 - 5 (like very much $=5$, like slightly $=4$, neutral $=3$, dislike slightly $=2$, dislike very much $=1$ ). The overall acceptable preference was on a scale of 1 - 2 (acceptable $=2$, not acceptable $=1$ ).

Data are presented as means \pm SEM (Table 4).

\section{Statistical Analysis}

Before accepting analysis of variance results, Lavene's test was used to check for homogeneity of variances among treatments. Since variances were considered homogenous from test results, analysis of variance was used to test for significant differences among treatments. Tukey's test for comparison of all possible pairs of means was used. SAS Proc GLM was used for all statistical analysis and testing [9] [10]. A value of $(P \leq 0.05)$ was considered the criterion of significance.

\section{Results and Discussion}

Taste panel evaluation by 64 in-house volunteers for the gluten-free ancient whole grain flatbreads is given in Table 4. Color/Appearance of quinoa and amaranth flatbreads were judged as similar and significantly $(\mathrm{P} \leq 0.05)$ higher than those of teff and buckwheat flatbreads. Values for teff flatbread were also significantly higher than those for buckwheat flatbread. The data suggest that lighter color flatbreads of quinoa and amaranth were preferred for Color/Appearance over dark colored teff and buckwheat, similarly brown color teff was favored over charcoal brown color buckwheat. Odor/Aroma as well as Texture/Mouth-feel evaluation for quinoa flatbread was significantly higher than other flatbreads tested. Values for amaranth and teff flatbreads were similar and significantly higher than those for buckwheat flatbread. Taste/Flavor for quinoa, amaranth and teff flatbreads were judged to be similar and significantly better than buckwheat flatbreads. Acceptance for quinoa and teff flat 
Table 2. Formulation of ancient whole grain gluten-free flatbread dough.

\begin{tabular}{|c|c|c|c|}
\hline Whole Grain & $\begin{array}{c}\text { Flour } \\
\mathrm{g}\end{array}$ & $\begin{array}{c}\text { Salt } \\
\mathrm{g}\end{array}$ & $\begin{array}{c}\text { Water } \\
\mathrm{mL}\end{array}$ \\
\hline Quinoa & 300 & 2.4 & 350 \\
\hline Teff & 250 & 2.4 & 325 \\
\hline Amaranth & 300 & 2.4 & 350 \\
\hline Buckwheat & 250 & 2.4 & 400 \\
\hline
\end{tabular}

Table 3. Composition of mint-cilantro-onion chutney.

\begin{tabular}{cc}
\hline Ingredients & $\mathrm{g} / \mathrm{mL}$ \\
\hline Mint Leaves (fresh) & $100 \mathrm{~g}$ \\
Cilentro (fresh) & $100 \mathrm{~g}$ \\
Red Onion & $340 \mathrm{~g}$ \\
Jalapeno Pepper (no seed core) & $60 \mathrm{~g}$ \\
Salt & $16 \mathrm{~g}$ \\
Lemon Juice (fresh) & $120 \mathrm{~mL}$ \\
\hline
\end{tabular}

Mint leaves, cilantro and jalapeno were blanched. Blended all ingredients to homogenous paste and stored at $4^{\circ} \mathrm{C}$.

Table 4. Results of taste panel sensory evaluation of ancient whole grain gluten-free flatbreads ${ }^{1,2}$.

\begin{tabular}{cccccc}
\hline Flatbread & Color/Appearance & Odor/Aroma & Taste/Flavor & Texture/Mouth Feel & Acceptance \\
\hline Quinoa & $4.61 \pm 0.07^{\mathrm{a}}$ & $4.25 \pm 0.11^{\mathrm{a}}$ & $3.67 \pm 0.14^{\mathrm{a}}$ & $4.09 \pm 0.10^{\mathrm{a}}$ & $1.84 \pm 0.05^{\mathrm{a}}$ \\
Teff & $3.27 \pm 0.13^{\mathrm{b}}$ & $3.66 \pm 0.11^{\mathrm{b}}$ & $3.58 \pm 0.11^{\mathrm{a}}$ & $3.44 \pm 0.13^{\mathrm{b}}$ & $1.72 \pm 0.06^{\mathrm{a}}$ \\
Amaranth & $4.33 \pm 0.10^{\mathrm{a}}$ & $3.72 \pm 0.13^{\mathrm{b}}$ & $3.31 \pm 0.17^{\mathrm{a}}$ & $3.34 \pm 0.16^{\mathrm{b}}$ & $1.66 \pm 0.06^{\mathrm{b}}$ \\
Buckwheat & $2.70 \pm 0.13^{\mathrm{c}}$ & $3.05 \pm 0.14^{\mathrm{c}}$ & $2.83 \pm 0.13^{\mathrm{b}}$ & $2.52 \pm 0.15^{\mathrm{c}}$ & $1.38 \pm 0.06^{\mathrm{c}}$ \\
\hline
\end{tabular}

${ }^{1}$ Values are mean \pm SEM; $\mathrm{n}=64$ bolor/Appearance, Odor/Aroma, Taste/Flavor and Texture/ Mouth feel were on a scale of $1-5$ (like very much $=5$, like slightly $=4$, neutral $=3$, dislike slightly $=2$ and dislike very much $=1)$; Acceptance was on scale of $1-2$ (acceptable $=2$ and unacceptable $=1$ ).

${ }^{2}$ Values within columns with different superscript letters differ significantly $(\mathrm{P} \leq 0.05)$.

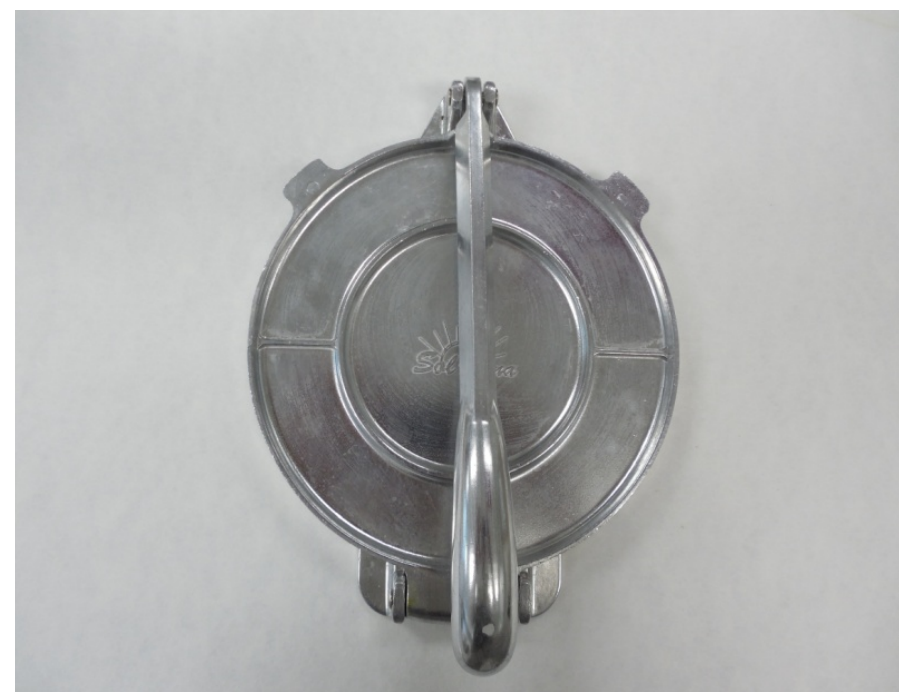

Figure 1. Sol luna $20 \mathrm{~cm}$ tortilla, flatbread press. 


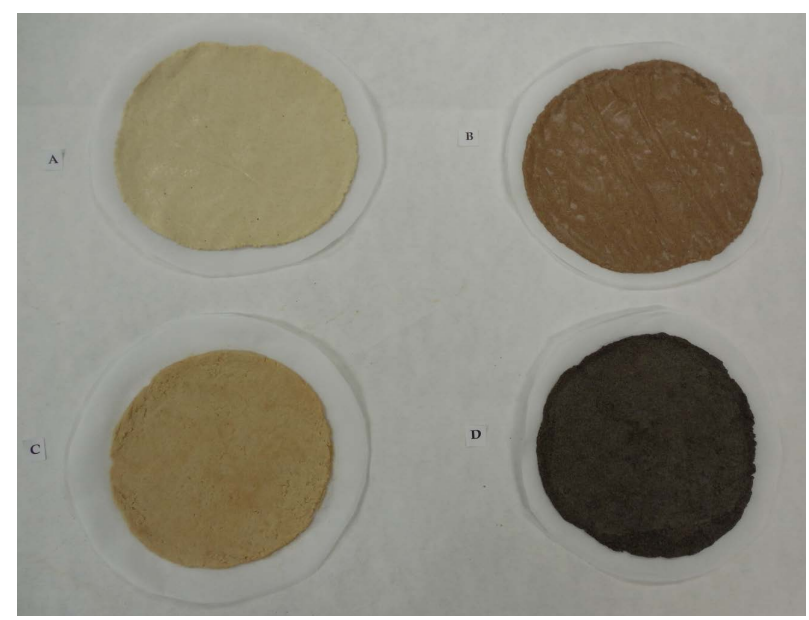

Figure 2. Uncooked ancient whole grain gluten-free flatbreads $\sim 17 \mathrm{~cm}$ circles, made in $20 \mathrm{~cm}$ sol luna tortilla flatbread press (Figure 1(a)). 65 g dough pressed between sheets of parchment paper (non-stick, oven safe); (A) quinoa; (B) teff; (C) amaranth; (D) buckwheat.

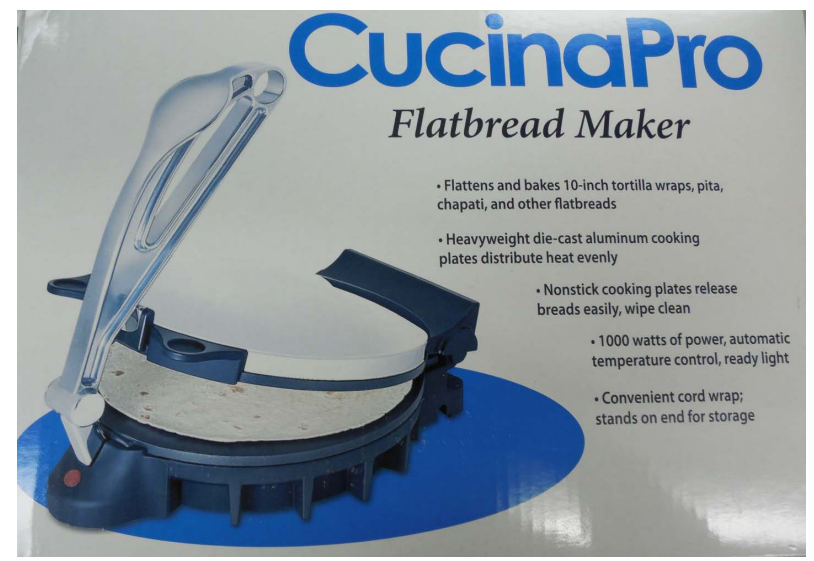

Figure 3. Cucina pro flatbread maker.

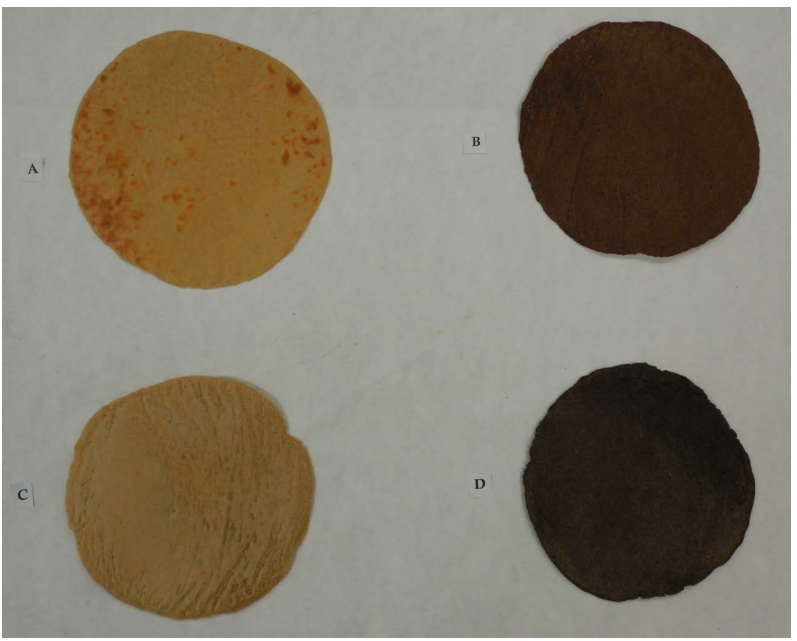

Figure 4. Cooked ancient whole grain gluten-free flatbreads, (A) quinoa; (B) teff; (C) amaranth; (D) buckwheat. 


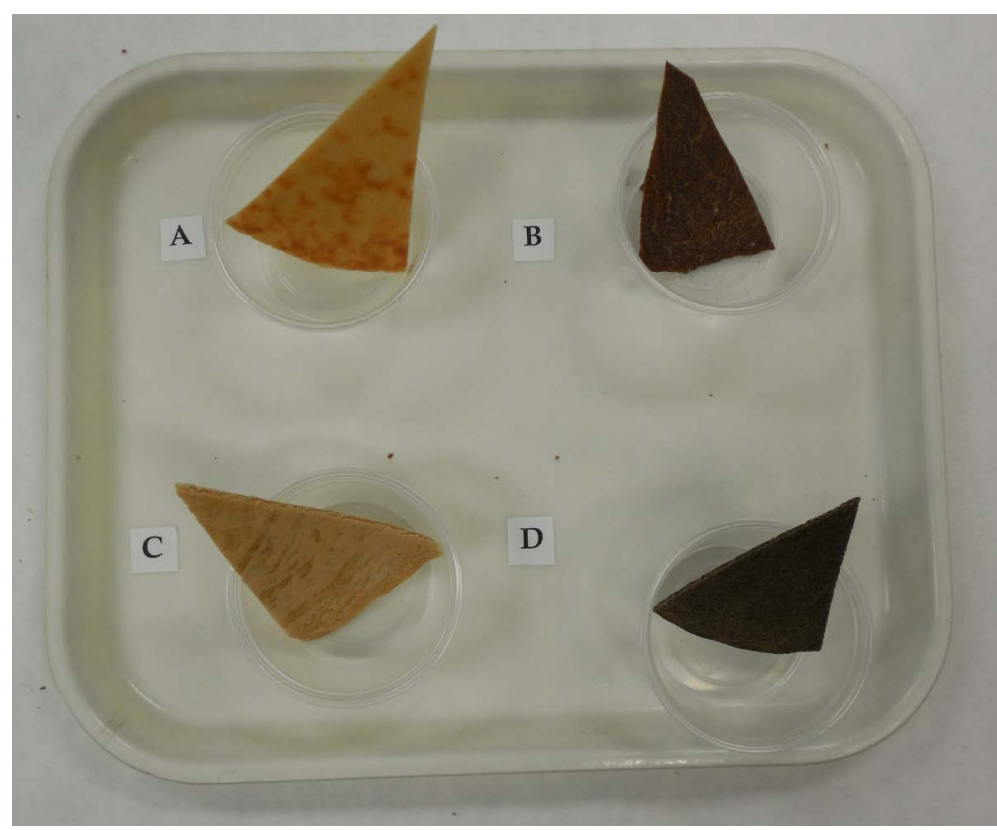

Figure 5. Taste panelists were offered ancient whole grain gluten-free flatbreads to taste as such or with chutney or mild salsa (Figure 6); (A) quinoa; (B) teff; (C) amaranth; and (D) buckwheat. Flatbreads were evaluated for Color/Appearance, Odor/Aroma, Taste/Flavor and Texture/Mouth feel were on a scale of 1 - 5 (like very much $=5$, like slightly $=4$, neutral $=3$, dislike slightly = 2 and dislike very much $=1$ ); Acceptance was on scale of 1 - 2 (acceptable $=2$ and unacceptable $=1$ ).

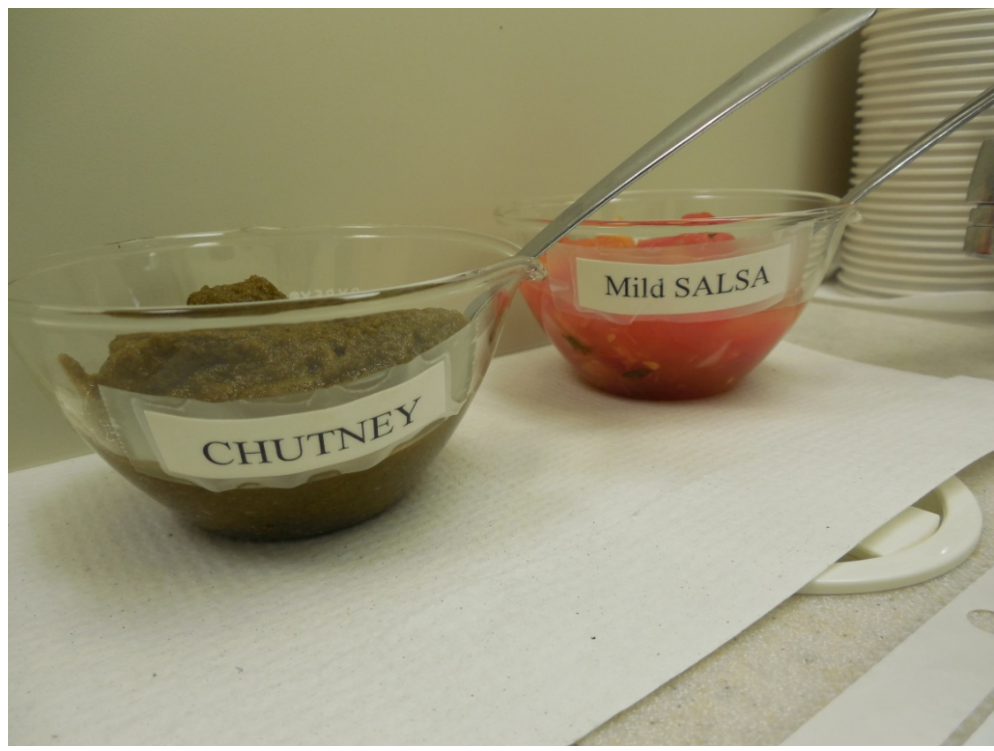

Figure 6. Mint-Cilantro-Onion Chutney was formulated and prepared in the laboratory kitchen, Chutney contained fresh mint leaves and cilantro $100 \mathrm{~g}$ each, jalapeno $60 \mathrm{~g}$, red onions $340 \mathrm{~g}$, salt $16 \mathrm{~g}$ and fresh lemon juice $120 \mathrm{~mL}$. Salsa (mild) obtained from refrigerator section of local grocery market.

breads was similar and significantly higher than amaranth and buckwheat flatbreads. Acceptance for amaranth flatbread was significantly higher than for buckwheat flatbread. Lower acceptance for amaranth than teff flatbreads is due to strong after taste of amaranth besides its preferred lighter Color/Appearance than that for teff. 
The panelists judged acceptance for ancient whole grain gluten-free flatbreads for quinoa $84 \%$, teff $72 \%$, amaranth 66\% and buckwheat 38\% (Figure 7). The acceptance for quinoa, teff and amaranth flatbreads was very encouraging.

Overall desirability of ancient whole grain gluten free flatbreads was calculated as percent sum of (like very much + like slightly + one-half of neutral). Overall desirability for quinoa, teff, amaranth and buckwheat flatbreads was for Color/Appearance (98\%, 59\%, 92\% and 38\%), for Odor/Aroma (88\%, 73\%, 75\% and 50\%), for Taste/Flavor (76\%, 73\%, 60\% and 43\%) and for Texture/Mouth-feel (89\%, 63\%, 59\% and 33\%), respectively. The data suggest that panelists found that ancient whole grain gluten-free (no yeast or chemicals) flatbreads of quinoa, teff and amaranth were very acceptable and offer good tasting healthy choice. Buckwheat flatbreads were judged with lowest score for all the sensory evaluation parameters. It may be due in part to its charcoal brown color, high dietary fiber content and/or gritty Texture/Mouth-feel with this ancient whole grain. Each ancient grain gluten-free flatbread contained 25 - $30 \mathrm{~g}$ whole grain and 4 - $5 \mathrm{~g}$ protein. Quinoa, teff and amaranth one flatbread contained 2 - $3 \mathrm{~g}$ dietary fiber, whereas each buckwheat flatbread contained $8 \mathrm{~g}$ of dietary fiber. Consuming four ancient whole grain quinoa, teff and amaranth flatbreads in two meals (2/meal) would provide 8 $12 \mathrm{~g}$ of dietary fiber, meeting nearly $30 \%-50 \%$ of the daily recommended level of dietary fiber intake. Buckwheat flat breads would meet total daily dietary fiber requirement, however its sensory evaluation parameters are needed to be improved. This is the only report demonstrating innovative ancient whole grain gluten-free health promoting products. These gluten-free flatbreads could be made in any house hold or commercial production. This study would help individuals to increase their whole grain consumption to meet the recommendations of the USDA Nutrition Policy guidelines 2010. This report documents the desirable acceptance of gluten-free ancient whole grain flatbreads for the first time. In addition it offers healthy and tasty choice of easy to make whole grain flatbreads for gluten sensitive individuals. The future studies would test formulations of buckwheat and its blend with other gluten-free whole grains with the expectation of more desirable sensory evaluation. Flatbread could be used to make sandwich, stuffed roll-ups or served on the side with a meal. Prepared dough may be stored in a refrigerator for several days, prepared uncooked flat breads may be frozen and cooked when desired to be served.

\section{Advantages of Gluten-Free Ancient Whole Grain Flat Breads}

We have elaborated on many reasons for the popularity of flatbreads described by Faridi [11]. Gluten-free ancient whole grain flatbreads (no yeast or chemicals) reported herein, offer healthy choices to all consumers and especially to those with sensitivity to gluten. Ancient whole grains flatbreads are made with $100 \%$ extraction flours. They are good sources of protein, dietary fiber, minerals and contain all the benefits of phytonutrients contained in the bran portion of the grains. The formulations of the flatbreads are simple as it contains only flour, salt and water. Even the salt could be eliminated due preference of lowering sodium intake. Flatbreads are ex-

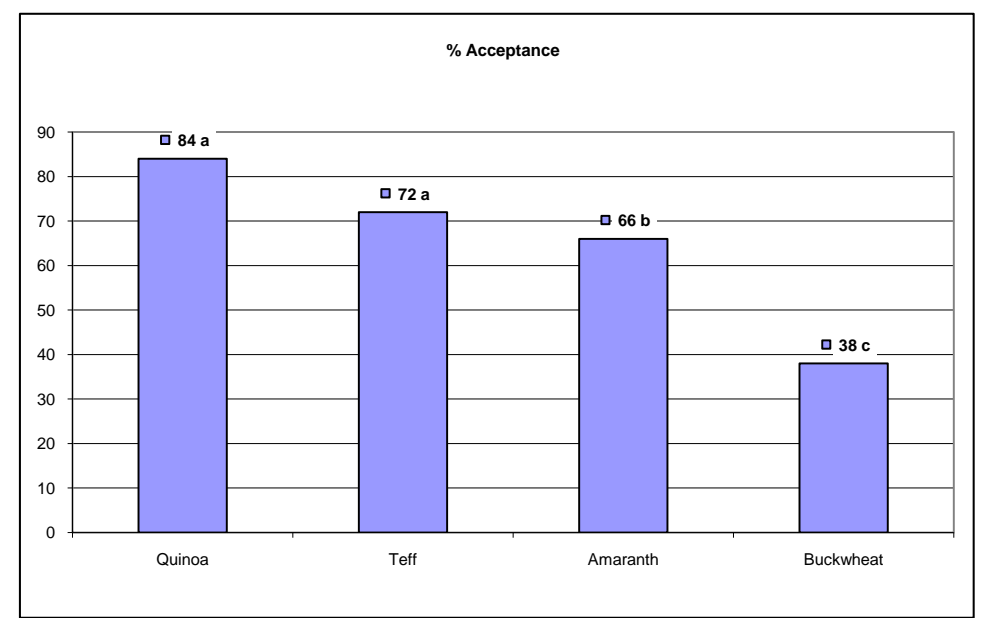

Figure 7. Percent taste panel acceptance of gluten-free quinoa, teff, amaranth and buckwheat flatbreads, $\mathrm{n}=64$. Bars with different superscript letters differ significantly $(\mathrm{P} \leq 0.05)$. 
cellent carriers by incorporating food ingredients such as meats, vegetables, condiments or spices in the dough or rolled in a single layer of flat breads or served on the side with curries, chutnies, soups, vegetables and meat dishes. The preparation is simple for any home or commercial production to make fresh ancient whole grain gluten-free flatbreads. The flatbreads offer real advantages for baking industry for their commercial marketing. As the ingredients are a few that offers significant reduction in cost and storage. Flatbreads can be processed directly on the conveyer belts, substantial savings could be recognized in equipment needs. Flat breads have lower volume for shipping and storage. The large surface area of the flat breads requires less time and space for cooling. Large number of products can be made with only slight modifications to the production line. Composition, nutritional and physiochemical properties of the cooked gluten free flatbreads may be studied for further characterization of these products. Flatbreads can be stored in several ways: Room temperature: 5 days; Refrigerated: 25 days; Frozen: 6 months.

\section{Conclusion}

Ancient whole grains make nutritious gluten-free flatbreads that are simple to make at home as well as in a commercial production. It can be every day staple for all families. It is low in fat, calories, good source of protein, fiber and minerals. Taste panels of 64 in-house volunteers determined that Taste/Flavor and overall acceptance for quinoa, teff and amaranth flatbreads very desirable. Novel health promoting ancient whole grain gluten-free flatbreads had quinoa $84 \%$, teff $72 \%$, amaranth $66 \%$ and buckwheat $38 \%$ acceptance. Each ancient whole grain flatbread contained 25 - $30 \mathrm{~g}$ whole grain and 4 - 5 g protein. Quinoa, teff and amaranth one flatbread contained 2 - 3 g dietary fiber, whereas buckwheat flatbread contained $8 \mathrm{~g}$ dietary fiber. Consuming two whole grain breads with two meals (total $=4$ ) would give 4 - $32 \mathrm{~g}$ of dietary fiber. Any house hold could make these breads. The gluten sensitive individuals would enjoy tasty, nutritious, ancient whole grain, gluten-free easy to make flatbreads. These recipes offer consumers additional healthy choices and would lead to increase in whole grain consumption. Nutritional and physiochemical characteristics of these ancient whole grain glutenfree flatbreads are warranted in future investigations.

\section{References}

[1] Qarooni, J., Ponte, J.G. and Posner, E.S. (1992) Flat Breads of the World. Cereal Foods World, 37, 863-865.

[2] USDA (2010) Center for Nutrition Policy and Promotion; Dietary Guidelines for Americans. http://www.choosemyplate.gov/

[3] Whole Grain Council (2009) http://wholegrainscouncil.org/whole-grains-101/what-are-the-health-benefits

[4] US Food and Drug Administration (2003) http://google2.fda.gov/search?q=whole+grain+claim\&client=FDAgov\&site=FDAgov\&lr=\&proxystylesheet=FDAgov \&requiredfields=-archive\%3AYes\&output=xml_no_dtd\&getfields=*

[5] US Food and Drug Administration (2013) http://www.fda.gov/downloads/ForConsumers/ConsumerUpdates/UCM363276.pdf

[6] Kahlon, T.S. and Chiu, M.M. (2012) Whole Grain Gluten-Free Flat Breads. Cereal Foods World, 57, 6-9. http://dx.doi.org/10.1094/CFW-57-1-0006

[7] AOAC (2000) Official Methods of Analysis of the Association of Official Analytical Chemists. 17th Edition, The Association, Arlington, Methods 985.29, 990.03.

[8] AOAC (1990) Official Methods of Analysis of the Association of Official Analytical Chemists. 15th Edition, The Association, Arlington, Methods 935.29, 942.05.

[9] Steel, R.G.D. and Torrie, J.H. (1960) Principles and Procedures of Statistics. McGraw-Hill, New York.

[10] SAS Institute Inc. (2004) SAS Online Doc 9.1.3. SAS Institute Inc., Cary, North Carolina.

[11] Faridi, H. (1981) Health Advantages of a High Bread Diet and Approaches to US-Type Flatbread Production. Bakers Digest, 55, 73-81. 
Scientific Research Publishing (SCIRP) is one of the largest Open Access journal publishers. It is currently publishing more than 200 open access, online, peer-reviewed journals covering a wide range of academic disciplines. SCIRP serves the worldwide academic communities and contributes to the progress and application of science with its publication.

Other selected journals from SCIRP are listed as below. Submit your manuscript to us via either submit@scirp.org or Online Submission Portal.
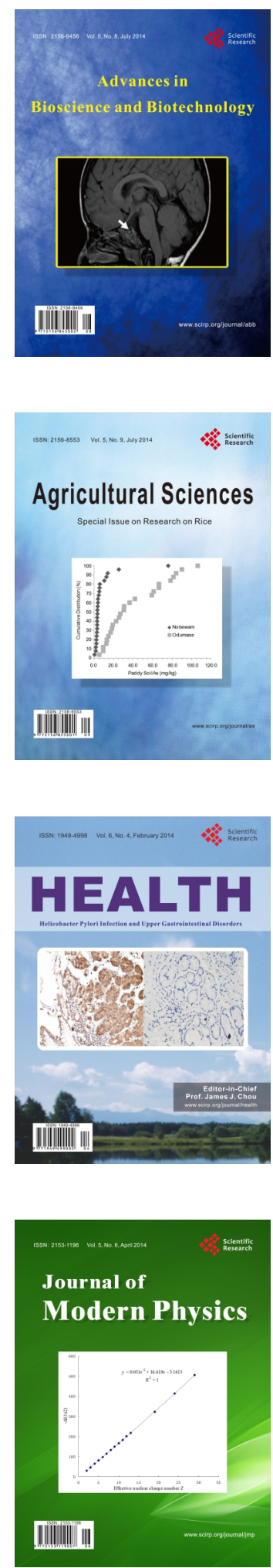
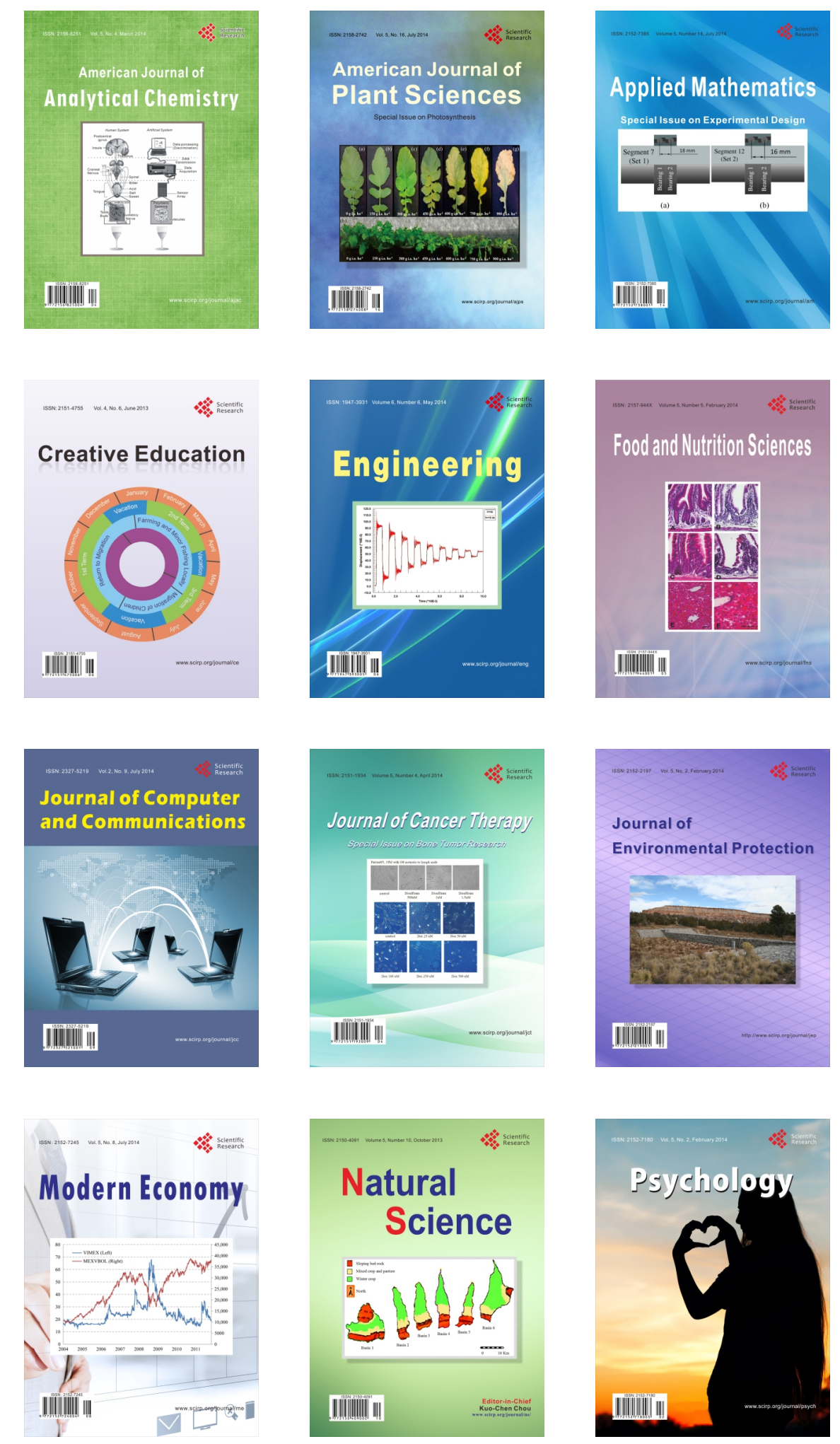